\title{
Primary autoimmune myelofibrosis with severe thrombocytopenia mimicking immune thrombocytopenia: A case report
}

\author{
JIAN HUA, SHU MATAYOSHI, TOMOYUKI UCHIDA, MORIHIRO INOUE and MASAO HAGIHARA \\ Department of Hematology, Eiju General Hospital, Tokyo 110-8645, Japan
}

Received July 26, 2016; Accepted October 24, 2016

DOI: $10.3892 / \mathrm{mco} .2016 .1064$

\begin{abstract}
Patients presenting with bone marrow fibrosis not accompanied by well-established autoimmune diseases, such as systemic lupus erythematosus, or malignant diseases, are considered to have primary autoimmune myelofibrosis (AIMF). Primary AIMF has been reported to follow a benign course and responds well to treatment with immunosuppressive agents. Immune thrombocytopenia (ITP) is also an autoimmune disorder characterized by antiplatelet-antibodymediated thrombocytopenia in the absence of other causes of thrombocytopenia. We herein present a rare case of a female patient who was diagnosed with primary AIMF. The patient presented with severe thrombocytopenia, which was initially misdiagnosed as ITP. The symptoms of the disease resolved completely following steroid treatment. After withdrawal of the treatment at 1 year from the diagnosis, the bone marrow examination showed no evidence of bone marrow fibrosis or other abnormalities. To date, the patient has been followed up for 2 years without evidence of disease.
\end{abstract}

\section{Introduction}

Autoimmune myelofibrosis (AIMF) is a benign disease that was first described in 1994 as a distinct clinicopathological entity associated with diffuse bone marrow fibrosis and autoimmune phenomena (1). AIMF is mainly encountered in patients with well-established autoimmune diseases, such as systemic lupus erythematosus (SLE). In patients without well-established autoimmune diseases, AIMF is defined as primary, characteristically follows a benign course and responds well to treatment with steroids and/or other immunosuppressive agents (1). Immune thrombocytopenia (ITP) is also an autoimmune disorder characterized by antiplatelet-antibody-mediated thrombocytopenia in the

Correspondence to: Dr Jian Hua, Department of Hematology, Eiju General Hospital, 2-23-16 Higashi-Ueno, Taito-ku, Tokyo 110-8645, Japan

E-mail: hhongshi1996@yahoo.co.jp

Key words: primary autoimmune myelofibrosis, immune thrombocytopenia, steroids absence of other causes of thrombocytopenia (2). Bone marrow fibrosis has also been reported in a proportion of ITP patients treated with thrombopoietin receptor agonists (3). Although low-grade bone marrow fibrosis [reticulin; grade 0-1 on the European Consensus scale (4)] has also been observed in newly diagnosed ITP patients, but fibrosis of grade $>1$ in such patients is very rare (5). We herein report a rare case of a patient with severe thrombocytopenia exhibiting grade 2 bone marrow fibrosis, who was diagnosed with primary AIMF initially misdiagnosed as ITP.

\section{Case report}

A 52-year-old female patient with a 5-year history of type 2 diabetes mellitus treated with voglibose presented to a local hospital with extensive petechiae on her legs. The patient was diagnosed with thrombocytopenia and referred to the Department of Hematology, Eiju General Hospital (Tokyo, Japan). A laboratory evaluation revealed pancytopenia with mild leukopenia and anemia, in addition to marked thrombocytopenia. The white blood cell count was $3.1 \times 10^{9} / 1$ (neutrophils: 48\%, monocytes: $6 \%$ and lymphocytes: $44 \%$ ), there were no atypical cells on the blood smear, the hemoglobin level was $10^{9} \mathrm{~g} / 1$ and the platelet count was $4 \times 10^{9} / 1$. The serum lactate dehydrogenase, immunoglobulin (Ig)G, platelet-associated IgG and rheumatoid factor levels were elevated to $251 \mathrm{U} / 1$ (normal, <223 U/1), $28.47 \mathrm{~g} / 1$ (normal, $<17 \mathrm{~g} / \mathrm{l}$ ), $438 \mathrm{ng} / 10^{7}$ cells (normal, $<46 \mathrm{ng} / 10^{7}$ cells), and $70 \mathrm{IU} / \mathrm{ml}$ (normal, $15 \mathrm{IU} / \mathrm{ml}$ ), respectively. In addition, the reticulated platelet count, plasma thrombopoietin level and plasma transforming growth factor $\beta$ (TGF- $\beta$ ) level were significantly elevated to $13 \%$ (normal, $<5 \%$ ), $3.4 \mathrm{fmol} / \mathrm{ml}$ (normal range, $0.48 \pm 0.28 \mathrm{fmol} / \mathrm{ml}$ ), and $23.7 \mathrm{ng} / \mathrm{ml}$ (normal, $<3.24 \mathrm{ng} / \mathrm{ml}$ ), respectively. The antinuclear antibody titer was within normal limits and no anti-GPIIb/IIIa antibody-producing B cells were detected in the serum. Tests for mutations in the Janus kinase-2 (JAK-2), thrombopoietin receptor (MPL) and calreticulin (CALR) genes were all negative. Bone marrow aspiration was attempted at the sternum and the left and right posterior inferior iliac spines; however, only a dry tap was obtained each time. Bone marrow biopsy from the left posterior inferior iliac spine revealed a hypercellular bone marrow (Fig. 1A) with high-grade fibrosis (grade 2 on the European Consensus scale; Fig. 1B) and an increase in the number of megakaryocytes (Fig. 1C). Abdominal 

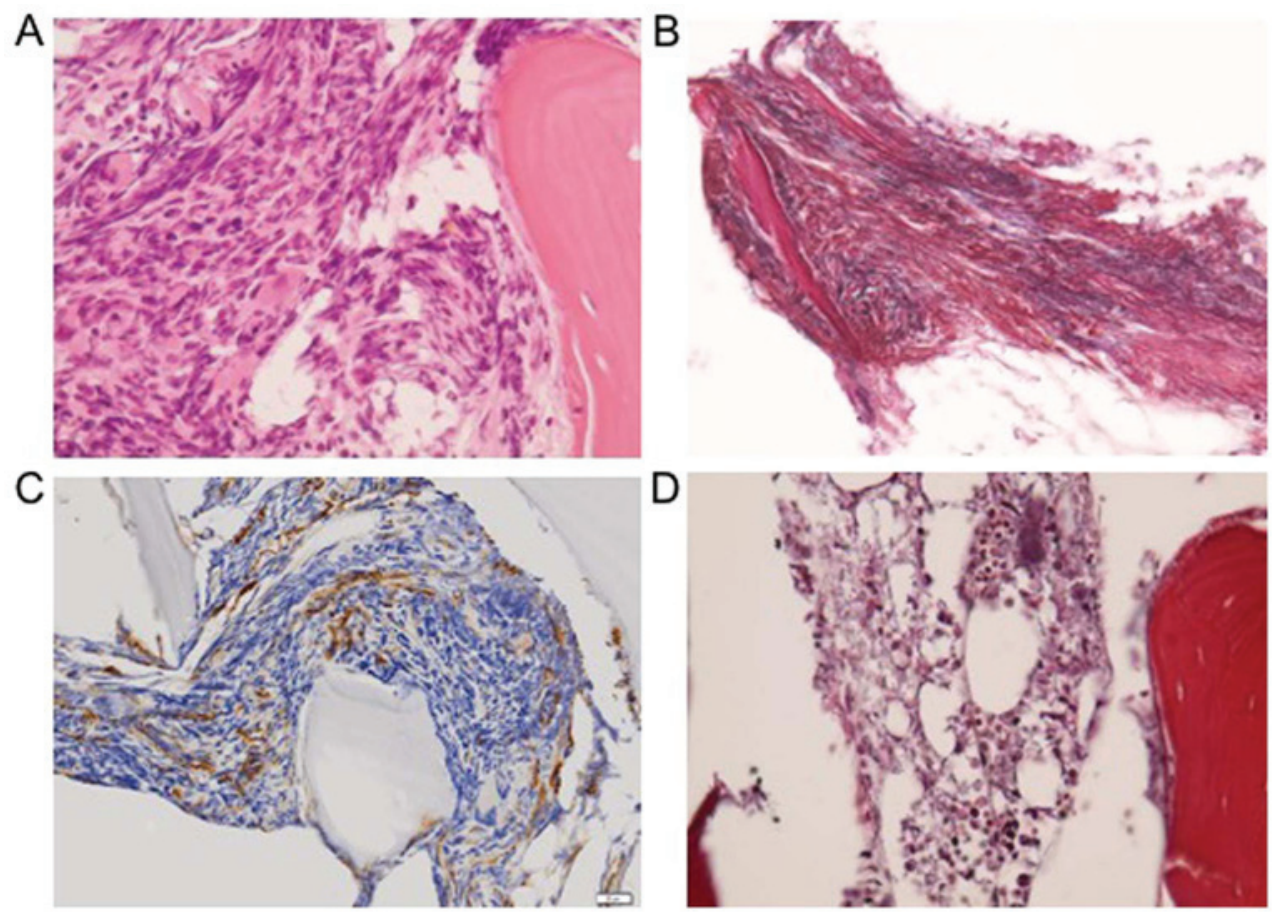

Figure 1. Bone marrow biopsies. (A) Hypercellular bone marrow on hematoxylin and eosin staining; (B) diffuse and dense increase in reticulin with extensive intersections (MF2) on silver staining; (C) increased megakaryocyte count as diagnosed by factor VIII staining at diagnosis; and (D) scattered linear reticulin with no intersections, corresponding to normal bone marrow (MF-0) following steroid treatment. Original magnification, $\mathrm{x} 40$ in all panels. MF, myelofibrosis.

computed tomography (CT) showed no splenomegaly or lymphadenopathy. On the basis of these results, the patient was diagnosed with ITP complicated by bone marrow fibrosis. After the patient received intravenous IgG treatment ( $400 \mathrm{mg} / \mathrm{kg}$ for 5 days) from day 2 after admission, the platelet count increased to $38 \times 10^{9} / 1$ on day 8 , slowly decreasing again thereafter to $3 \times 10^{9} / 1$ on day 24 . On the same day (day 24), the patient complained of headache and dizziness, and was diagnosed with left cerebellar hemorrhage on head CT (Fig. 2). Intravenous $\mathrm{IgG}$ treatment was repeated at the same doses, followed by oral administration of prednisolone at $1 \mathrm{mg} / \mathrm{kg}$ per day. Thereafter, the platelet count promptly increased to normal within 7 days, and the patient was discharged on day 66 after admission. The dose of prednisolone was slowly tapered, and the treatment was discontinued at 1 year from the diagnosis, while the patient was followed up on an outpatient basis. A bone marrow aspirate from the left posterior inferior iliac spine was repeated after complete withdrawal of the prednisolone and revealed no abnormalities, and a repeat bone marrow biopsy at 1 year from the diagnosis revealed no evidence of bone marrow fibrosis (Fig. 1D). To date, the patient has been followed up for 2 years without evidence of disease. The patient provided written informed consent regarding the publication of the case details.

\section{Discussion}

Myelofibrosis, encountered in association with various benign and malignant disorders, is characterized by reticulin fibrosis of the bone marrow with resultant myelophthisis. Primary idiopathic myelofibrosis is well-known as a clonal Philadelphia chromosome-negative myeloproliferative

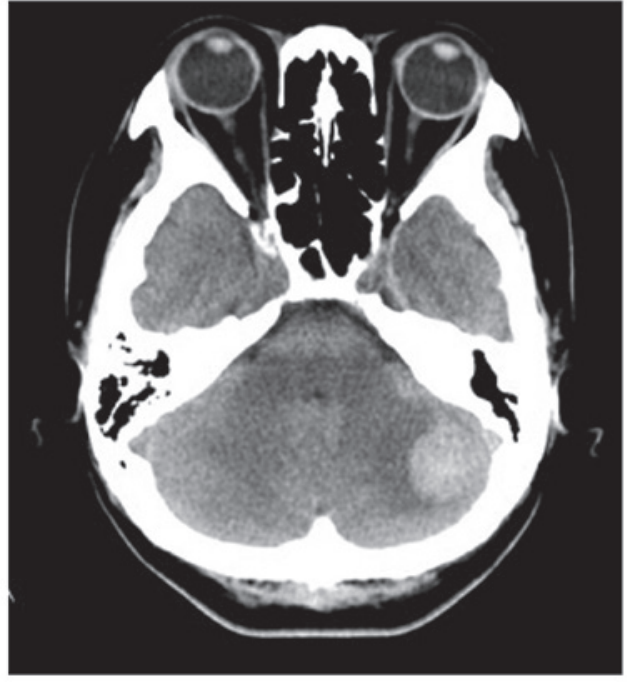

Figure 2. Computed tomography image of the head showing a left cerebellar hemorrhage.

neoplasm characterized by extramedullary hematopoiesis and marrow fibrosis, with a proportion of the patients harboring mutations of the JAK-2, MPL or CALR genes (6). Secondary myelofibrosis may occur in various neoplastic disorders, such as chronic myeloid leukemia, acute megakaryoblastic leukemia, malignant lymphoma, myelodysplastic syndrome and bone marrow metastasis (7). In addition to these malignant causes, AIMF has also been described in association with well-established autoimmune disorders, particularly SLE (1). Myelofibrosis encountered in cases without a well-established autoimmune disorder or malignant disease is defined as 
primary AIMF (8). The diagnosis of primary AIMF is based on the presence of reticulin fibrosis and lymphocyte infiltration of the bone marrow, presence of autoantibodies (e.g., anti-nuclear antibody and rheumatoid factor) in the serum, and absence of clustered or atypical megakaryocytes, myeloid or erythroid dysplasia, eosinophilia or basophilia, splenomegaly, or any other disorder known to cause myelofibrosis (8). Although there was a mild increase of the rheumatoid factor titer in the serum of our patient, there were no symptoms, such as arthralgia, suggestive of any of the well-established autoimmune disorders. On the basis of the abovementioned criteria, the patient was diagnosed with primary AIMF.

It was previously reported that primary AIMF shows a favorable response to corticosteroid therapy (1). In our case, prednisolone was initiated at $1 \mathrm{mg} / \mathrm{kg} /$ day and tapered over a 3-month period, achieving complete normalization of the blood counts. Furthermore, a follow-up bone marrow biopsy, performed after the steroid treatment, revealed complete resolution of the bone marrow fibrosis. The efficacy of the steroid treatment in our case was consistent with previous reports $(1,8)$.

It has been suggested that certain cytokines, such as the fibrogenic cytokine TGF- $\beta$, the angiogenic cytokine basic fibroblast growth factor, and tissue inhibitors of metalloproteinase, may be involved in the development of bone marrow fibrosis (9). Furthermore, megakaryocytes and activated monocytes are considered to be important sources of TGF- $\beta$, which induces fibroblast proliferation (10). Our patient exhibited an increase of the TGF- $\beta$ concentration in the serum and of the megakaryocyte counts in biopsy samples at diagnosis, with normalization of the serum TGF- $\beta$ (data not shown) after steroid treatment. These observations support the hypothesis that TGF- $\beta$ generated by megakaryocytes is the most likely cause of marrow fibrosis in AIMF.

Due to the severe thrombocytopenia in our patient, the diagnosis of ITP was initially considered on admission. An increased number of anti-GPIIb/IIIa antibody-producing B cells and an elevated percentage of reticulated platelets in the peripheral blood, as well as a normal or mildly increased plasma thrombopoietin level in ITP cases have been previously reported, which are recognized as useful adjuncts in the diagnosis of ITP (11). However, our patient did not exhibit any increase in the number of anti-GPIIb/IIIa antibody-producing $\mathrm{B}$ cells in the blood, although an elevation in the percentage of reticulated platelets and mild increase of the plasma thrombopoietin level were observed. A mild increase in the marrow reticulin content (grade 0-1 on the European Consensus scale) has also been reported in untreated adult ITP patients, as compared to the general adult population (5). Furthermore, bone marrow fibrosis has been reported in some ITP patients treated with thrombopoietin receptor agonists (3). However, marrow reticulin fibrosis of grade $>1$ is very rare in primary ITP. Moreover, our patient also exhibited a mildly elevated serum rheumatoid factor titer, mild leukopenia and anemia, all of which are well known to occur in AIMF patients (8), in addition to the severe thrombocytopenia. Thus, the clinical characteristics in our patient were more consistent with the diagnosis of primary AIMF rather than ITP.

In conclusion, we herein present a rare case of a female patient who was diagnosed with primary AIMF characterized by severe thrombocytopenia, which was similar to the findings in ITP. The disease symptoms, including pancytopenia and marrow fibrosis, responded well to glucocorticoid therapy, with complete remission. To evaluate the pathological mechanisms underlying this disease, a larger number of cases must be accumulated in the future.

\section{Acknowledgements}

The authors would like to thank Dr Masaya Akatsuka and Dr Masayuki Shimoda for providing the histopathological findings and images, and Dr Hiroshi Yamaguchi for determining the mutation status of the JAK-2, MPL and CALR genes.

\section{References}

1. Pullarkat V, Bass RD, Gong JZ, Feinstein DI and Brynes RK: Primary autoimmune myelofibrosis: Definition of a distinct clinicopathologic syndrome. Am J Hematol 72: 8-12, 2003.

2. McKenzie CG, Guo L, Freedman J and Semple JW: Cellular immune dysfunction in immune thrombocytopenia (ITP). $\mathrm{Br}$ J Haematol 163: 10-23, 2013.

3. Brynes RK, Orazi A, Theodore D, Burgess P, Bailey CK, Thein MM and Bakshi KK: Evaluation of bone marrow reticulin in patients with chronic immune thrombocytopenia treated with eltrombopag: Data from the EXTEND study. Am J Hematol 90: 598-601, 2015.

4. Thiele J, Kvasnicka HM, Facchetti F, Franco V, van der Walt J and Orazi A: European consensus on grading bone marrow fibrosis and assessment of cellularity. Haematologica 90: 1128-1132, 2005.

5. Rizvi H, Butler T, Calaminici M, Doobaree IU, Nandigam RC, Bennett D, Provan D and Newland AC: United Kingdom immune thrombocytopenia registry: Retrospective evaluation of bone marrow fibrosis in adult patients with primary immune thrombocytopenia and correlation with clinical findings. Br J Haematol 169: 590-594, 2015.

6. Tefferi A: Myeloproliferative neoplasms: A decade of discoveries and treatment advances. Am J Hematol 91: 50-58, 2016.

7. Gianelli U, Vener C, Bossi A, Cortinovis I, Iurlo A, Fracchiolla NS, Savi F, Moro A, Grifoni F, De Philippis C, et al: The European Consensus on grading of bone marrow fibrosis allows a better prognostication of patients with primary myelofibrosis. Mod Pathol 25: 1193-1202, 2012.

8. Vergara-Lluri ME, Piatek CI, Pullarkat V, Siddiqi IN, O'Connell C, Feinstein DI and Brynes RK: Autoimmune myelofibrosis: An update on morphologic features in 29 cases and review of the literature. Hum Pathol 45: 2183-2191, 2014.

9. Tefferi A: Pathogenesis of myelofibrosis with myeloid metaplasia. J Clin Oncol 23: 8520-8530, 2005.

10. Kuter DJ, Bain B, Mufti G, Bagg A and Hasserjian RP: Bone marrow fibrosis: Pathophysiology and clinical significance of increased bone marrow stromal fibres. Br J Haematol 139: 351-362, 2007.

11. Kuwana M, Okazaki Y, Satoh T, Asahi A, Kajihara M and Ikeda Y: Initial laboratory findings useful for predicting the diagnosis of idiopathic thrombocytopenic purpura. Am J Med 118: 1026-1033, 2005. 